\title{
Prevalence and determinants of diabetes and prediabetes in southwestern Iran: the Khuzestan comprehensive health study (KCHS)
}

Sanam Hariri ${ }^{1 \dagger}$, Zahra Rahimi ${ }^{2 \dagger}$, Nahid Hashemi-Madani ${ }^{3}$, Seyyed Ali Mard ${ }^{4}$, Farnaz Hashemi ${ }^{1}$, Zahra Mohammadi ${ }^{1}$, Leila Danehchin ${ }^{5}$, Farhad Abolnezhadian ${ }^{6,7}$, Aliasghar Valipour ${ }^{8}$, Yousef Paridar ${ }^{9}$, Mohammad Mahdi Mir-Nasseri ${ }^{10}$, Alireza Khajavi ${ }^{11}$, Sahar Masoudi ${ }^{1}$, Saba Alvand ${ }^{1}$, Bahman Cheraghian ${ }^{12}$, Ali Akbar Shayesteh ${ }^{13}$, Mohammad E. Khamseh ${ }^{3^{*}}$ and Hossein Poustchi ${ }^{1^{*}}$

Background: The Middle East and North Africa (MENA) is postulated to have the highest increase in the prevalence of diabetes by 2030; however, studies on the epidemiology of diabetes are rather limited across the region, including in Iran.

Methods: This study was conducted between 2016 and 2018 among Iranian adults aged 20 to 65 years residing in Khuzestan province, southwestern Iran. Diabetes was defined as the fasting blood glucose (FBG) level of $126 \mathrm{mg} / \mathrm{dl}$ or higher, and/or taking antidiabetic medications, and/or self-declared diabetes. Prediabetes was defined as FBG 100 to $125 \mathrm{mg} / \mathrm{dl}$. Multinomial logistic regression models were used to examine the association of multiple risk factors that attained significance on the outcome.

Results: Overall, 30,498 participants were recruited; the mean $( \pm$ SD) age was $41.6( \pm 11.9)$ years. The prevalence of prediabetes and diabetes were 30.8 and $15.3 \%$, respectively. We found a similar prevalence of diabetes in both sexes, although it was higher among illiterates, urban residents, married people, and smokers. Participants aged 5065 and those with Body Mass Index (BMI) $30 \mathrm{~kg} / \mathrm{m}^{2}$ or higher were more likely to be affected by diabetes [RR: 20.5 $(18.1,23.3)$ and $3.2(3.0,3.6)]$. Hypertension [RR: $5.1(4.7,5.5)]$, waist circumference (WC) equal or more than $90 \mathrm{~cm}$ [RR: $3.6(3.3,3.9)]$, and family history [RR: $2.3(2.2,2.5)]$ were also significantly associated with diabetes. For prediabetes, the main risk factors were age 50 to 65 years [RR: $2.6(2.4,2.8)]$, BMl $30 \mathrm{~kg} / \mathrm{m}^{2}$ or higher [RR: $\left.1.9(1.8,2.0)\right]$, hypertension and WC of $90 \mathrm{~cm}$ or higher [RR: $1.7(1.6,1.8)]$. The adjusted relative risks for all variables were higher in females than males, with the exception of family history for both conditions and waist circumference for prediabetes.

\footnotetext{
*Correspondence: khamseh.m@iums.ac.ir; h.poustchi@gmail.com

+Sanam Hariri and Zahra Rahimi contributed equally to this work.

${ }^{3}$ Endocrine Research Center, Institute of Endocrinology and Metabolism, Iran

University of Medical Sciences, Tehran, Iran

${ }^{1}$ Liver and Pancreatobiliary Diseases Research Center, Digestive Diseases

Research Institute, Shariati Hospital, Tehran University of Medical Sciences, N.

Kargar St, Tehran, Iran

Full list of author information is available at the end of the article
}

(c) The Author(s). 2021 Open Access This article is licensed under a Creative Commons Attribution 4.0 International License, which permits use, sharing, adaptation, distribution and reproduction in any medium or format, as long as you give appropriate credit to the original author(s) and the source, provide a link to the Creative Commons licence, and indicate if changes were made. The images or other third party material in this article are included in the article's Creative Commons licence, unless indicated otherwise in a credit line to the material. If material is not included in the article's Creative Commons licence and your intended use is not permitted by statutory regulation or exceeds the permitted use, you will need to obtain permission directly from the copyright holder. To view a copy of this licence, visit http://creativecommons.org/licenses/by/4.0/ The Creative Commons Public Domain Dedication waiver (http://creativecommons.org/publicdomain/zero/1.0/) applies to the data made available in this article, unless otherwise stated in a credit line to the data. 
Conclusions: Prediabetes and diabetes are prevalent in southwestern Iran. The major determinants are older age, obesity, and the presence of hypertension. Further interventions are required to escalate diabetes prevention and diagnosis in high-risk areas across Iran.

Keywords: Diabetes, Prediabetes, Epidemiology, Risk-factors, Low and middle-income countries, Middle-east, Iran

\section{Background}

The Middle East and North Africa (MENA) region holds the highest prevalence of diabetes mellitus (DM) worldwide, and is estimated to have the most significant increase in diabetes prevalence in the coming years [1]. However, there are limited population-based studies on the epidemiology and determinants of diabetes in MENA countries, including Iran [2].

By 2035, the number of adults with diabetes will increase by $55 \%$ globally, and the greatest proportional increase is expected in low-income, followed by low to middle-income countries [3]. The MENA region is estimated to have an over $95 \%$ increase in the number of people with diabetes by 2035 [4]. Nearly half of all diabetes deaths in this region occur among people under 60 years old; these premature deaths may be due to the new emerging risk factors, delayed diagnoses, and weak health-care systems that are not competent to bear the growing burden [5]. In addition, the absence of focused preventive measures, as well as lack of sufficient health insurance coverage, are among the major reasons underlying the high diabetes burden in MENA [6]. Aging populations, together with rapid urbanization that has brought major behavioral and lifestyle changes in many countries in this region, have resulted in a dramatic increase in diabetes prevalence [7]. Identifying the main traditional and non-traditional diabetes risk factors is crucial for developing preventive strategies and early diagnosis in such countries [2].

In Iran, the number of people with diabetes has been projected to increase by $91 \%$, and its economic burden will increase markedly by $2035[8,9]$. Despite the availability of medications and insulin coverage in Iran, utilization of antidiabetic drugs and control of blood glucose remains subpar [10-12]. According to previous national reports, only $13 \%$ of diabetes patients in Iran have controlled hyperglycemia [12]. Another Iranian study among patients living in urban areas reported that over one-third have hemoglobin A1c (HbA1c) levels higher than 9\% [13]. In line with global initiatives to reduce the diabetes burden, Iran has made great prevention efforts in recent decades [14-16]. However, the rising trend of diabetes is outpacing such efforts and current role of diabetes risk factors remains poorly identified specially in remote areas across Iran [2, 12]. The present study aimed to investigate the prevalence and determinants of prediabetes and diabetes in a large population from southwestern Iran.

\section{Methods}

Study design

The Khuzestan comprehensive health study (KCHS) was conducted as a large cross-sectional study between October 2016 and November 2018 to assess the health conditions of 30,500 Iranian adults. The study site was Khuzestan province, located in southwestern Iran, which covers an area of more than $63,000 \mathrm{~km}^{2}$ and is home to a diverse population of nearly five million, including Fars, Arab, Bakhtiari, Kurd, and Lur ethnic groups. Written informed consent was obtained from all participants. All procedures performed in the study were in accordance with the ethical guidelines of the Declaration of Helsinki and the ethics committee of the National Institute for Medical Research Development (NIMAD) has approved the study protocol. The KCHS methodology has been detailed elsewhere [17].

\section{Study population}

The study population includes all men and women aged 20 to 65 years, residing in urban and rural areas of Khuzestan province. The sample size required to estimate the prevalence of any health conditions in the province with $1 \%$ margin of error was calculated, and a $50 \%$ baseline prevalence to maximize the required sample size was assumed. Therefore, a sample size of 30,000 was chosen, which will provide a power greater than $90 \%$. To obtain a representative sample, the population of all 27 counties in the province were stratified and a random sample of primary care centers -called health houses- in each county were chosen, which was proportional to total province population living in that county. Ultimately, 1079 random health houses were chosen and 30 individuals were randomly selected from the population covered by each center. Inclusion criteria were having been resided in Khuzestan for a minimum of 1 year prior to the study enrolment. Exclusion criteria were unwillingness to participate and any physical or psychological disability that hinder the participation.

\section{Study procedures}

A multistage random sampling method was used to select participants. Trained staff went door to door to explain the study goals and invite the participants to the 
pre-defined study sites on the next day. The study sites include health centers, health houses, schools, and mosques, located in the close proximity of participants' residential address. All participants provided written informed consent upon registration.

Fifteen $\mathrm{mL}$ of blood were obtained from each individual, divided into one clot tube and one EDTA tube. Blood was transferred to the reference lab within three hours of sampling, stored in Cool boxes at $4{ }^{\circ} \mathrm{C}$. Blood samples were centrifuged at $3000 \mathrm{rpm}$ for $10 \mathrm{~min}$ to separate serum; thereafter, serum level of fasting blood glucose (FBG) was measured by BT 1500 AutoAnalyzer, using commercial kits (Pars Azmoon, Iran). In case of any abnormal laboratory results, participants were referred to family physicians for further evaluation.

To reduce the measurement error, anthropometric data were gathered after blood sample collection, in a fasting state for 8 to $12 \mathrm{~h}$. Height and weight were measured by the Seca 206 body meter measuring tape with Wall Stop and the Seca 762 mechanical flat scale, respectively, and the Seca 203 circumference measuring tape was used to measure waist circumference (WC). Blood pressure was measured using aneroid sphygmomanometers (Riester, Germany), twice from each arm in the sitting position. All measurements were in accordance with the US National Institutes of Health protocols. Data on demographics, socioeconomic status, and lifestyle habits were collected afterward by staffadministered questionnaires.

\section{Definition of variables and study outcomes}

Diabetes was defined as having an FBG level of $126 \mathrm{mg} /$ $\mathrm{dl}$ or higher, and/or taking antidiabetic medications, and/or self-declared diabetes. Prediabetes was defined as the FBG level of 100 or higher, but equal to or less than $125 \mathrm{mg} / \mathrm{dl}$. General obesity was determined based on Body mass index (BMI), which is calculated by dividing weight $(\mathrm{kg})$ by the square of the height $(\mathrm{m})$ and categorized according to the World Health Organization (WHO) cut-off points [18]. BMI scores less than $25 \mathrm{~kg} /$ $\mathrm{m}^{2}$, between 25 and $29.9 \mathrm{~kg} / \mathrm{m}^{2}$, and $30 \mathrm{~kg} / \mathrm{m}^{2}$ or higher were defined as normal weight, overweight, and obese, respectively. Abdominal obesity was characterized by a WC of $90 \mathrm{~cm}$ or higher for both men and women [19]. Following major guidelines, hypertension (HTN) was defined as systolic blood pressure (SBP) equal to or higher than $140 \mathrm{mmHg}$, and/or diastolic blood pressure (DBP) equal to or higher than $90 \mathrm{mmHg}$, and/or taking antihypertensive medications [20]. Wealth score index was calculated using multiple correspondence analysis (MCA) with the following variables: house area, freezer, washing machine, dishwasher, laptop, internet, car type, TV type, indoor bathroom, vacuum cleaner, mobile use, and foreign travels [21].

\section{Statistical analysis}

Data are described by the mean and standard deviation (SD) or frequency and percentage, where appropriate. A multinomial logistic regression model was used to assess the impact of the covariates on the risk of prediabetes or diabetes, which led to relative risks (RR) as the measure of association. In this model, individuals with prediabetes and diabetes were compared with normal participants. Each of the covariates sex, age, BMI, WC, HTN, family history of diabetes, and education have been put in the model, once alone and once adjusted for cigarette smoking (never/ever), hookah smoking (never/ever), opium use (never/ever), alcohol use (never/ever), marital status (single/married), residence (urban/rural), ethnicity (Arab/Bakhtiari/Fars/Turk/Kurd/Lur), and wealth index (four quartiles). These eight variables were selected as the adjustments since the preliminary assessments showed that the prevalence of prediabetes and diabetes did not differentiate between these variables' strata. Two separate regression models were created to identify factors associated with prediabetes and diabetes in men and women. The analyses were performed using the statistical software Stata (version 12), and the chosen significance level was 0.05 .

\section{Results}

Overall, 30,498 individuals were included in the analysis, of whom 19,600 (64.3\%) were women. The mean age of participants was $41.6 \pm 11.9$ years. The mean BMI and WC were $27.6 \pm 5.3 \mathrm{~kg} / \mathrm{m}^{2}$ and $92.5 \pm 13.4 \mathrm{~cm}$, respectively. Overall, the majority were married (83.0\%), urban residents (73.1\%), of Arab ethnicity (49.0\%), and had primary education (44.2\%). Eighty-nine percent were neversmokers, and cigarette smoking was reported among 28.0 and $1.3 \%$ of men and women, respectively. History of hookah and opium use was reported among 5.2 and $2.8 \%$, respectively. Family history of diabetes was reported in $34.2 \%$ of all participants, and $19.7 \%$ were found to have HTN.

Among all participants, 9395 (30.8\%) were identified to have prediabetes, and 4673 (15.3\%) had diabetes. Diabetes had been self-reported among 2694 (57.7\%) individuals, and 1941 (41.5\%) had undiagnosed diabetes. The highest prevalence of diabetes was observed among people with HTN (33.5\%), those who aged 50-65 years (32.3\%), illiterates $(24.7 \%)$, and those with a family history of diabetes $(22.6 \%)$. For prediabetes, the highest prevalence was observed in people with BMI $30 \mathrm{~kg} / \mathrm{m}^{2}$ or higher $(34.6 \%)$, those with WC equal or more than $90 \mathrm{~cm}(33.2 \%)$, and the age range of $50-65(32.9 \%)$ or $35-49.9$ years (32.7\%). Prevalence of diabetes was similar in both sexes (15.3\%), while prediabetes was slightly higher in men (32.0 vs. 30.2\%). Both conditions were more prevalent among urban residents and marrieds. 
Table 1 Characteristics of the Khuzestan Comprehensive Health Study (KCHS) participants

\begin{tabular}{|c|c|c|c|c|}
\hline \multicolumn{2}{|l|}{ Variable } & \multirow{2}{*}{$\begin{array}{l}\text { Normal } \\
16,430\end{array}$} & \multirow{2}{*}{$\begin{array}{l}\text { Prediabetes } \\
9395\end{array}$} & \multirow{2}{*}{$\begin{array}{l}\text { Diabetes } \\
4673\end{array}$} \\
\hline Number & & & & \\
\hline \multirow[t]{2}{*}{ Sex } & Male & $5750(52.7 \%)$ & $3483(32.0 \%)$ & $1665(15.3 \%)$ \\
\hline & Female & $10,680(54.5 \%)$ & $5912(30.2 \%)$ & $3008(15.3 \%)$ \\
\hline \multirow[t]{3}{*}{ Age (years) } & $20-34.9$ & $7154(69.6 \%)$ & $2766(26.9 \%)$ & $353(3.5 \%)$ \\
\hline & $35-49.9$ & $6297(53.9 \%)$ & 3811 (32.7\%) & $1568(13.4 \%)$ \\
\hline & $50-65$ & $2950(34.8 \%)$ & 2797 (32.9\%) & $2745(32.3 \%)$ \\
\hline \multirow[t]{3}{*}{$\mathrm{BMI}\left(\mathrm{Kg} / \mathrm{m}^{2}\right)$} & $<25$ & $6343(64.5 \%)$ & 2593 (26.4\%) & $893(9.1 \%)$ \\
\hline & $25-29.9$ & $5991(52.4 \%)$ & $3616(31.6 \%)$ & $1833(16.0 \%)$ \\
\hline & $\geq 30$ & $4024(44.3 \%)$ & $3149(34.6 \%)$ & 1915 (21.1\%) \\
\hline \multirow[t]{2}{*}{ Waist circumference $(\mathrm{cm})$} & $<90$ & $8052(64.9 \%)$ & $3404(27.4 \%)$ & $949(7.7 \%)$ \\
\hline & $\geq 90$ & $8306(46.3 \%)$ & 5955 (33.2\%) & $3692(20.5 \%)$ \\
\hline \multirow[t]{2}{*}{ Hypertension } & No & $14,333(58.5 \%)$ & 7501 (30.6\%) & $2659(10.9 \%)$ \\
\hline & Yes & 2089 (34.9\%) & 1891 (31.6\%) & $2013(33.5 \%)$ \\
\hline \multirow[t]{2}{*}{ Diabetes family history } & No & $11,531(57.6 \%)$ & $6180(30.9 \%)$ & $2314(11.5 \%)$ \\
\hline & Yes & $4867(46.7 \%)$ & 3201 (30.7\%) & $2350(22.6 \%)$ \\
\hline \multirow[t]{4}{*}{ Education } & Illiterate & $2996(45.3 \%)$ & 1981 (30.0\%) & $1629(24.7 \%)$ \\
\hline & Elementary & $7094(52.6 \%)$ & 4300 (31.9\%) & $2086(15.5 \%)$ \\
\hline & Secondary & $4696(59.9 \%)$ & $2340(29.8 \%)$ & $808(10.3 \%)$ \\
\hline & Higher education & $1642(64.1 \%)$ & 772 (30.1\%) & $149(5.8 \%)$ \\
\hline \multirow[t]{2}{*}{ Cigarette smoking } & Never & $14,560(53.8 \%)$ & 8401 (31.1\%) & 4085 (15.1\%) \\
\hline & Ever & $1784(54.1 \%)$ & $950(28.8 \%)$ & $564(17.1 \%)$ \\
\hline \multirow[t]{2}{*}{ Hookah smoking } & Never & 15,435 (53.7\%) & 8853 (30.8\%) & 4461 (15.5\%) \\
\hline & Ever & 909 (57.1\%) & 489 (30.7\%) & 195 (12.2\%) \\
\hline \multirow[t]{2}{*}{ Opium use } & Never & $15,871(53.8 \%)$ & 9119 (30.9\%) & 4515 (15.3\%) \\
\hline & Ever & 489 (57.5\%) & 229 (26.9\%) & $133(15.6 \%)$ \\
\hline \multirow[t]{2}{*}{ Alcohol use } & Never & $15,936(53.7 \%)$ & 9135 (30.8\%) & 4589 (15.5\%) \\
\hline & Ever & 406 (62.7\%) & 186 (28.7\%) & $56(8.6 \%)$ \\
\hline \multirow[t]{2}{*}{ Marital status } & Single & 3050 (58.9\%) & 1559 (30.1\%) & $569(11.0 \%)$ \\
\hline & Married & 13,380 (52.8\%) & 7836 (31.0\%) & $4104(16.2 \%)$ \\
\hline \multirow[t]{4}{*}{ Ethnicity } & Arab & 7454 (49.9\%) & 4971 (33.3\%) & $2501(16.8 \%)$ \\
\hline & Bakhtiari & 4236 (62.9\%) & 1668 (24.8\%) & $831(12.3 \%)$ \\
\hline & Fars & 2984 (53.1\%) & 1726 (30.7\%) & $911(16.2 \%)$ \\
\hline & Turk/Kurd/Lur & $1720(54.6 \%)$ & 1010 (32.0\%) & $422(13.4 \%)$ \\
\hline \multirow[t]{2}{*}{ Residence } & Urban & $11,605(52.1 \%)$ & 7001 (31.4\%) & 3678 (16.5\%) \\
\hline & Rural & 4824 (58.7\%) & 2394 (29.2\%) & 995 (12.1\%) \\
\hline \multirow[t]{4}{*}{ Wealth index } & $\mathrm{Q}_{1}$ & 4239 (55.5\%) & $2271(29.7 \%)$ & 1131 (14.8\%) \\
\hline & $\mathrm{Q}_{2}$ & 4018 (53.4\%) & $2340(31.1 \%)$ & 1161 (15.5\%) \\
\hline & $\mathrm{Q}_{3}$ & 4435 (54.8\%) & 2457 (30.4\%) & 1197 (14.8\%) \\
\hline & $\mathrm{Q}_{4}$ & 3641 (51.5\%) & 2274 (32.2\%) & $1152(16.3 \%)$ \\
\hline
\end{tabular}

People who belonged to the highest wealth index category and those of the Arab ethnicity had the highest prevalence for both diabetes (16.3 and 16.8\%) and prediabetes (32.2 and $33.3 \%)$, respectively (Table 1$)$. The standardized prevalence that was calculated according to the demographics, including sex, age, education, and BMI strata, did not change compared to the unadjusted prevalence of both conditions.

Results on multivariate analysis of the factors associated with diabetes and prediabetes risk are demonstrated 
in Table 2. For diabetes, the most significant risk factors include older age [RR (95\% CI); 20.54 (18.13, 23.28)], HTN [RR (95\%CI); 5.09 (4.72, 5.49)], and a WC of $90 \mathrm{~cm}$ or higher [RR (95\% CI); 3.62 (3.34, 3.92)]. Moreover, compared to the participants with a BMI less than $25 \mathrm{~kg} / \mathrm{m}^{2}$, those who had BMI 25-29.9 $\mathrm{kg} / \mathrm{m}^{2}$ [RR (95\% CI); $\left.2.11(1.93,2.31)\right]$ or BMI equal to or higher than $30 \mathrm{~kg} / \mathrm{m}^{2}$ [RR (95\% CI); $3.24(2.96$, 3.56)] were more likely to be affected by diabetes. Having a history of diabetes in first-degree family member was associated with a two-fold higher likelihood of having diabetes [RR $(95 \% \mathrm{CI}) ; 2.30$ (2.15, 2.46)], while higher education was associated with a lower risk [RR (95\% CI); $0.10(0.09,0.13)]$.

Regarding prediabetes, the most significant risk factors were older age [RR $(95 \% \mathrm{CI}) ; 2.61(2.43,2.81)]$, BMI equal to $30 \mathrm{~kg} / \mathrm{m}^{2}$ or higher [RR $(95 \% \mathrm{CI}) ; 1.88(1.76$, $2.01)]$, HTN [RR $(95 \% \mathrm{CI}) ; 1.71(1.60,1.83)]$, and a WC of $90 \mathrm{~cm}$ or more [RR $(95 \% \mathrm{CI}) ; 1.66(1.58,1.76)]$. Similar to diabetes, the highest educational degree was associated with a lower risk of prediabetes [RR $(95 \% \mathrm{CI}) ; 0.61$ $(0.54,0.68)]$. Furthermore, female sex was slightly protective against prediabetes [(RR $(95 \% \mathrm{CI}) ; 0.87 \quad(0.82$, 0.92)] (Table 2).
Results on multivariate analysis of the investigated factors stratified by gender are presented in Tables 3 and 4. We observed a statistically significant difference for the association of diabetes and age among genders; the relative risk was more than two-fold higher for women with 50-65 years of age, compared to men in the same age range [RR: 25.02 vs. 11.56].

Among women, HTN had a higher relative risk for diabetes [RR: 6.46 vs. 3.00] and prediabetes [RR: 1.91 vs. 1.37] compared to men. Similarly, abdominal obesity in women was more significantly associated with diabetes [RR: 3.90 vs. 2.85]. Women with abdominal and general obesity had a similar likelihood of having diabetes [RR: 3.90 vs. 3.93], while among men, abdominal obesity had a higher relative risk for diabetes [RR: 2.85 vs. 2.47]. The relative risk for diabetes family history was slightly higher among men [RR: 2.49 vs. 2.25]. Regarding socioeconomic status, women with higher education had a lower relative risk for diabetes compared to men [RR: 0.03 vs. 0.27$]$.

For prediabetes, the most significant risk factors were older age and general obesity between both men [RR: 1.82 and 1.72] and women [RR: 3.01 and 2.04], respectively, and older women had significantly higher relative

Table 2 Univariate and multivariate analysis of risk factors associated with diabetes and prediabetes in the Khuzestan study

\begin{tabular}{|c|c|c|c|c|c|}
\hline \multicolumn{2}{|l|}{ Variable } & \multicolumn{2}{|l|}{ Prediabetes } & \multicolumn{2}{|l|}{ Diabetes } \\
\hline Sex & Male & $\begin{array}{l}\text { Unadjusted. RR } \\
(95 \% \mathrm{Cl})\end{array}$ & $\begin{array}{l}\text { Adjusted. } \mathrm{RR}^{*} \\
(95 \% \mathrm{Cl})\end{array}$ & $\begin{array}{l}\text { Unadjusted. RR } \\
(95 \% \mathrm{Cl})\end{array}$ & $\begin{array}{l}\text { Adjusted. RR } \\
(95 \% \mathrm{Cl})\end{array}$ \\
\hline & Female & $0.91(0.87,0.96)$ & $0.87(0.82,0.92)$ & $0.97(0.91,1.04)$ & $0.98(0.91,1.06)$ \\
\hline \multirow[t]{3}{*}{ Age (years) } & $20-34.9$ & & & & \\
\hline & $35-49.9$ & $1.57(1.47,1.66)$ & $1.63(1.53,1.73)$ & $5.05(4.47,5.69)$ & $5.25(4.63,5.95)$ \\
\hline & $50-65$ & $2.45(2.29,2.62)$ & $2.61(2.43,2.81)$ & $18.86(16.75,21.24)$ & $20.54(18.13,23.28)$ \\
\hline \multirow[t]{3}{*}{ BMI $\left(\mathrm{Kg} / \mathrm{m}^{2}\right)$} & $<25$ & & & & \\
\hline & $25-29.9$ & $1.48(1.39,1.57)$ & $1.47(1.38,1.56)$ & $2.17(1.99,2.37)$ & $2.11(1.93,2.31)$ \\
\hline & $\geq 30$ & $1.91(1.79,2.04)$ & $1.88(1.76,2.01)$ & $3.38(3.09,3.69)$ & $3.24(2.96,3.56)$ \\
\hline \multirow[t]{2}{*}{ Waist circumference $(\mathrm{cm})$} & $<90$ & & & & \\
\hline & $\geq 90$ & $1.70(1.61,1.79)$ & $1.66(1.58,1.76)$ & $3.77(3.49,4.08)$ & $3.62(3.34,3.92)$ \\
\hline \multirow[t]{2}{*}{ Hypertension } & No & & & & \\
\hline & Yes & $1.73(1.62,1.85)$ & $1.71(1.60,1.83)$ & $5.19(4.82,5.59)$ & $5.09(4.72,5.49)$ \\
\hline \multirow[t]{2}{*}{ Diabetes family history } & No & & & & \\
\hline & Yes & $1.23(1.16,1.30)$ & $1.17(1.11,1.24)$ & $2.41(2.25,2.57)$ & $2.30(2.15,2.46)$ \\
\hline \multirow[t]{4}{*}{ Education } & Illiterate & & & & \\
\hline & Elementary & $0.92(0.86,0.98)$ & $0.86(0.80,0.92)$ & $0.54(0.50,0.58)$ & $0.43(0.40,0.47)$ \\
\hline & Secondary & $0.75(0.70,0.81)$ & $0.67(0.62,0.73)$ & $0.32(0.29,0.35)$ & $0.21(0.19,0.24)$ \\
\hline & Higher education & $0.71(0.64,0.79)$ & $0.61(0.54,0.68)$ & $0.17(0.14,0.20)$ & $0.10(0.09,0.13)$ \\
\hline
\end{tabular}

*Data are adjusted for cigarette smoking (never vs. ever), hookah smoking (never vs. ever), opium use (never vs. ever), alcohol use (never vs. ever), marital status (single vs. married), residence (urban vs. rural), ethnicity (Arab vs. Bakhtiari vs. Fars vs. Turk/Kurd/Lur), and wealth index (four quartiles) 
Table 3 Univariate and multivariate analysis of risk factors associated with diabetes and prediabetes among women in the Khuzestan study

\begin{tabular}{|c|c|c|c|c|c|}
\hline \multirow[t]{2}{*}{ Variables } & & \multicolumn{2}{|l|}{ Prediabetes } & \multicolumn{2}{|l|}{ Diabetes } \\
\hline & & $\begin{array}{l}\text { Unadjusted. RR } \\
(95 \% \mathrm{Cl})\end{array}$ & $\begin{array}{l}\text { Adjusted. } \mathrm{RR}^{*} \\
(95 \% \mathrm{Cl})\end{array}$ & $\begin{array}{l}\text { Unadjusted. RR } \\
(95 \% \mathrm{Cl})\end{array}$ & $\begin{array}{l}\text { Adjusted. } \mathrm{RR}^{*} \\
(95 \% \mathrm{Cl})\end{array}$ \\
\hline \multirow[t]{3}{*}{ Age (years) } & $20-34.9$ & & & & \\
\hline & $35-49.9$ & $1.64(1.52,1.76)$ & $1.69(1.56,1.82)$ & $5.62(4.83,6.54)$ & $5.83(4.99,6.81)$ \\
\hline & $50-65$ & $2.85(2.61,3.10)$ & $3.01(2.75,3.29)$ & $23.2(19.94,26.99)$ & $25.02(21.42,29.22)$ \\
\hline \multirow[t]{3}{*}{$\mathrm{BMI}\left(\mathrm{Kg} / \mathrm{m}^{2}\right)$} & $<25$ & & & & \\
\hline & $25-29.9$ & $1.44(1.32,1.56)$ & $1.47(1.36,1.60)$ & $2.43(2.16,2.73)$ & $2.47(2.18,2.79)$ \\
\hline & $\geq 30$ & $1.99(1.84,2.16)$ & $2.04(1.88,2.22)$ & $3.86(3.44,4.34)$ & $3.93(3.48,4.43)$ \\
\hline \multirow{2}{*}{$\begin{array}{l}\text { Waist circumference } \\
(\mathrm{cm})\end{array}$} & $<90$ & & & & \\
\hline & $\geq 90$ & $1.62(1.52,1.73)$ & $1.63(1.53,1.74)$ & $3.87(3.51,4.25)$ & $3.90(3.54,4.31)$ \\
\hline \multirow[t]{2}{*}{ Hypertension } & No & & & & \\
\hline & Yes & $1.91(1.75,2.08)$ & $1.91(1.74,2.08)$ & $6.54(5.95,7.18)$ & $6.46(5.87,7.10)$ \\
\hline \multirow{2}{*}{$\begin{array}{l}\text { Diabetes } \\
\text { family history }\end{array}$} & No & & & & \\
\hline & Yes & $1.22(1.14,1.30)$ & $1.17(1.09,1.25)$ & $2.31(2.12,2.51)$ & $2.25(2.07,2.44)$ \\
\hline \multirow[t]{4}{*}{ Education } & Illiterate & & & & \\
\hline & Elementary & $0.87(0.80,0.94)$ & $0.82(0.75,0.89)$ & $0.49(0.45,0.54)$ & $0.41(0.37,0.45)$ \\
\hline & Secondary & $0.66(0.60,0.73)$ & $0.59(0.53,0.66)$ & $0.23(0.21,0.27)$ & $0.16(0.14,0.18)$ \\
\hline & Higher education & $0.58(0.51,0.66)$ & $0.49(0.43,0.57)$ & $0.05(0.03,0.07)$ & $0.03(0.02,0.05)$ \\
\hline
\end{tabular}

"Data are adjusted for cigarette smoking (never vs. ever), hookah smoking (never vs. ever), opium use (never vs. ever), alcohol use (never vs. ever), marital status (single vs. married), residence (urban vs. rural), ethnicity (Arab vs. Bakhtiari vs. Fars vs. Turk/Kurd/Lur), and wealth index (four quartiles)

"RR Relative risk

Table 4 Univariate and multivariate analysis of risk factors associated with diabetes and prediabetes among men in the Khuzestan study

\begin{tabular}{|c|c|c|c|c|c|}
\hline \multirow[t]{2}{*}{ Variables } & & \multicolumn{2}{|l|}{ Prediabetes } & \multicolumn{2}{|l|}{ Diabetes } \\
\hline & & $\begin{array}{l}\text { Unadjusted. RR } \\
(95 \% \mathrm{Cl})\end{array}$ & $\begin{array}{l}\text { Adjusted. } \mathrm{RR}^{*} \\
(95 \% \mathrm{Cl})\end{array}$ & $\begin{array}{l}\text { Unadjusted. RR } \\
(95 \% \mathrm{Cl})\end{array}$ & $\begin{array}{l}\text { Adjusted. RR* } \\
(95 \% \mathrm{Cl})\end{array}$ \\
\hline \multirow[t]{3}{*}{ Age (years) } & $20-34.9$ & & & & \\
\hline & $35-49.9$ & $1.44(1.30,1.59)$ & $1.38(1.23,1.55)$ & $4.13(3.38,5.03)$ & $3.56(2.85,4.46)$ \\
\hline & $50-65$ & $1.92(1.72,2.14)$ & $1.82(1.61,2.07)$ & $13.42(11.08,16.27)$ & $11.56(9.24,14.47)$ \\
\hline \multirow[t]{3}{*}{$\mathrm{BMI}\left(\mathrm{Kg} / \mathrm{m}^{2}\right)$} & $<25$ & & & & \\
\hline & $25-29.9$ & $1.57(1.42,1.72)$ & $1.46(1.32,1.61)$ & $1.94(1.71,2.22)$ & $1.66(1.45,1.90)$ \\
\hline & $\geq 30$ & $1.88(1.68,2.12)$ & $1.72(1.52,1.94)$ & $3.02(2.60,3.50)$ & $2.47(2.11,2.88)$ \\
\hline \multirow{2}{*}{$\begin{array}{l}\text { Waist circumference } \\
(\mathrm{cm})\end{array}$} & $<90$ & & & & \\
\hline & $\geq 90$ & $1.83(1.68,2.00)$ & $1.66(1.51,1.82)$ & $3.61(3.16,4.12)$ & $2.85(2.48,3.27)$ \\
\hline \multirow[t]{2}{*}{ Hypertension } & No & & & & \\
\hline & Yes & $1.47(1.32,1.64)$ & $1.37(1.22,1.53)$ & $3.48(3.08,3.93)$ & $3.00(2.65,3.41)$ \\
\hline \multirow{2}{*}{$\begin{array}{l}\text { Diabetes } \\
\text { family history }\end{array}$} & No & & & & \\
\hline & Yes & $1.28(1.16,1.40)$ & $1.23(1.12,1.36)$ & $2.65(2.37,2.97)$ & $2.49(2.21,2.80)$ \\
\hline \multirow[t]{4}{*}{ Education } & Illiterate & & & & \\
\hline & Elementary & $1.05(0.92,1.21)$ & $0.97(0.84,1.12)$ & $0.69(0.59,0.81)$ & $0.55(0.47,0.65)$ \\
\hline & Secondary & $0.94(0.82,1.08)$ & $0.85(0.73,1.00)$ & $0.50(0.42,0.59)$ & $0.37(0.31,0.45)$ \\
\hline & Higher education & $0.97(0.81,1.15)$ & $0.84(0.69,1.02)$ & $0.40(0.32,0.51)$ & $0.27(0.21,0.36)$ \\
\hline
\end{tabular}

*Data are adjusted for cigarette smoking (never vs. ever), hookah smoking (never vs. ever), opium use (never vs. ever), alcohol use (never vs. ever), marital status (single vs. married), residence (urban vs. rural), ethnicity (Arab vs. Bakhtiari vs. Fars vs. Turk/Kurd/Lur), and wealth index (four quartiles) ${ }^{*} R R$ Relative risk 
Table 5 Characteristics of the Khuzestan study participants with diabetes who had Body Mass Index (BMI) less than $25 \mathrm{~kg} / \mathrm{m} 2$

\begin{tabular}{|c|c|c|}
\hline Variable & & Number (\%) \\
\hline People with diabetes and $\mathrm{BMI}<25 \mathrm{~kg} / \mathrm{m} 2$ & & 893 \\
\hline \multirow[t]{2}{*}{ Sex } & Male & $445(49.8 \%)$ \\
\hline & Female & $448(50.2 \%)$ \\
\hline \multirow[t]{3}{*}{ Age (years) } & 20-34.9 & $99(11.1 \%)$ \\
\hline & $35-49.9$ & $248(27.8 \%)$ \\
\hline & $50-65$ & $545(61.1 \%)$ \\
\hline \multirow[t]{2}{*}{ Waist circumference (cm) } & $<90$ & $561(62.8 \%)$ \\
\hline & $\geq 90$ & $332(37.2 \%)$ \\
\hline \multirow[t]{2}{*}{ Hypertension } & No & $574(64.3 \%)$ \\
\hline & Yes & $319(35.7 \%)$ \\
\hline \multirow[t]{2}{*}{ Diabetes family history } & No & $463(51.9 \%)$ \\
\hline & Yes & $429(48.1 \%)$ \\
\hline \multirow[t]{4}{*}{ Education } & Illiterate & $309(34.6 \%)$ \\
\hline & Elementary & $396(44.4 \%)$ \\
\hline & Secondary & $161(18.1 \%)$ \\
\hline & Higher education & $26(2.9 \%)$ \\
\hline \multirow[t]{2}{*}{ Cigarette smoking } & Never & $725(82.1 \%)$ \\
\hline & Ever & $158(17.9 \%)$ \\
\hline \multirow[t]{2}{*}{ Hookah smoking } & Never & $843(94.7 \%)$ \\
\hline & Ever & $47(5.3 \%)$ \\
\hline \multirow[t]{2}{*}{ Opium use } & Never & $842(94.7 \%)$ \\
\hline & Ever & 47 (5.3\%) \\
\hline \multirow[t]{2}{*}{ Alcohol use } & Never & $869(97.6 \%)$ \\
\hline & Ever & $2(2.4 \%)$ \\
\hline \multirow[t]{2}{*}{ Marital Status } & Single & $127(14.3 \%)$ \\
\hline & Married & $760(85.7 \%)$ \\
\hline \multirow[t]{2}{*}{ Residence } & Urban & $637(71.8 \%)$ \\
\hline & Rural & $250(28.2 \%)$ \\
\hline \multirow[t]{4}{*}{ Wealth index } & $\mathrm{Q}_{1}$ & $265(29.9 \%)$ \\
\hline & $\mathrm{Q}_{2}$ & $228(25.7 \%)$ \\
\hline & $\mathrm{Q}_{3}$ & $223(25.2 \%)$ \\
\hline & $\mathrm{Q}_{4}$ & $170(19.2 \%)$ \\
\hline
\end{tabular}

risk than men of the same age. No significant difference was observed for the association of abdominal obesity and prediabetes among both genders (Tables 3 and4).

We further evaluated the characteristics of the normal weight and underweight participants affected by diabetes (Table 5). Overall, 893 individuals with BMI less than $25 \mathrm{~kg} /$ $\mathrm{m}^{2}$ were identified to have diabetes; the mean age was $50.4 \pm 10.8$ years and $50.2 \%$ were female. The mean WC in this group was $86.1 \pm 8.8 \mathrm{~cm}$, mostly had a WC less than 90 $\mathrm{cm}$ (62.8\%). The majority aged 50-65 years (61.1\%), were married (85.7\%) and were urban residents (71.8\%). Most of these individuals had no history of HTN (64.3\%) and smoking $(82.1 \%)$, while nearly half had a family history of diabetes
(48.1\%) and one-third belonged to the lowest wealth index category (29.9\%). (Table 5).

\section{Discussion}

This study investigates the prevalence and determinants of prediabetes and diabetes in a population-based sample of Iranian adults residing in southwestern Iran. Prediabetes with $30.8 \%$ and diabetes with $15.3 \%$ were more prevalent in this area than in most other parts of the country. In line with some previous Iranian studies, older age, hypertension, and obesity were the most significant risk factors for diabetes and prediabetes [22] [23]. Also, family history of diabetes was associated with 
a two-fold higher risk of diabetes. These findings indicate that urgent control measures are needed to impede the high burden of diabetes in this region of Iran.

The prevalence of diabetes in this study was identical to a survey conducted by Yazdanpanah et al. in 2015, which is the only available report on diabetes from southwestern Iran (15.3\% vs. 15.1\%) [24], and was comparable to two recent studies from central (16.1\%) [25] and Northern Iran (17.2\%) [9]. However, this prevalence was nearly two-fold higher compared to the national estimations [26] and other parts of the country, including western [27], southeastern [28], and southern areas [29]. For prediabetes, two population-based studies in central and southeastern Iran have reported a lower prevalence (26 and 19\%); however, in both studies, individuals under the age of 35 years were also recruited [25]. These comparisons indicate that diabetes is exceptionally prevalent in the southwestern region of Iran, and urgent interventions should be taken to scale-up focused preventive and diagnostic programs [15]. Also, people of Arab ethnicity had the highest prevalence of both conditions. Given the subpar control of hyperglycemia in Iran, therapeutic interventions should be particularly expanded into the most high-risk populations and ethnic groups [12].

Globally, elderly people are at substantial risk for diabetes [30]. In this study, one-third of individuals aged $50-65$ years had diabetes, which is similar to the results of an elderly-based survey conducted among people above 60 years in southern Iran; however, their reported prevalence for prediabetes was two-fold higher than our estimation for elderlies (67\% vs. 33\%) [31]. Although older age was also the most significant risk factor for prediabetes, its relative risk was much lower compared to diabetes (2.4 vs. 18.5). Besides, older women had more than two-fold higher the relative risk of diabetes than men across the age range (25.0 vs. 11.6$)$, which can be explained by the large sex differences in obesity rates, especially after the age of 45 years and in countries with greater gender inequality [32, 33].

The second significant diabetes risk factor was HTN, having a five-fold higher relative risk in people with HTN than those without such condition, and a two-fold higher relative risk for women compared to men. This observation is in line with previous global reports [34]. Regarding obesity, some studies have emphasized the benefits of WC compared to BMI in predicting diabetes in the Iranian population $[1,35]$. In our study, WC showed a stronger association than BMI for diabetes (3.6 vs. 3.2), but not for prediabetes (1.7 vs. 1.9). Existing evidence has shown that men are diagnosed with diabetes at lower BMI than women of the same age group [36]. Accordingly, we observed no difference between WC and BMI among women for the risk of diabetes; however, BMI had a lower relative risk in men.

A study from Northern Iran showed that having one or more than one family member with diabetes increases the odds of this disease to 2.3 and 4.2, respectively [9]. Another cohort from the center of Iran showed that people with a first-degree family history were 3.3 times more likely to have the disease [37]. Similarly, family history in first-degree relatives, having a relative risk equal to 2.4 , was among the significant risk factors for diabetes in our study, with a slightly higher relative risk in men. Also, from people with diabetes and normal BMI, approximately half had a family history of diabetes, and the majority were from the lower socioeconomic categories. In a study conducted on over 9000 Iranian adults, Mirzaei et al. have found a negative interaction between family history of diabetes and other risk factors only for BMI [38]. Their results suggested that despite considering family history as an independent risk factor, as long as it is used as a tool to raise awareness and promote lifestyle changes, it would reduce the risk of developing diabetes in people with other risk factors such as obesity.

Evidence on the modifying effect of sex in the development of diabetes is controversial. Several Iranian studies have reported a higher prevalence among females $[1,39,40]$, and some others have not found any significant associations for sex [41]. Similar to the study of Yazdanpanah et al. from southwestern Iran, we observed a similar prevalence of diabetes in both sexes. Moreover, in our study, the female sex was slightly protective against prediabetes. According to a recent study, obesity is the most significant risk factor for sex inequalities in type 2 diabetes in Iranians and can partly explain the observed differences in the prevalence of diabetes between sexes [42].

Many studies have confirmed the role of socioeconomic factors in developing diabetes [43]. We observed higher diabetes and prediabetes prevalence among urban residents and illiterates. In fact, any level of education, mostly higher education, appears to be a protective factor for both diabetes and prediabetes. We also observed that higher education is more protective for diabetes among women, compared to men. In total, $8.4 \%$ of participants have had higher education, while only $2.9 \%$ of participants with diabetes and BMI $<25$ reported higher education. This observation suggests that lower education has bidirectional effects, both on its association with $\mathrm{BMI}<25$, and with higher prevalence of diabetes. These findings were in line with other studies on socioeconomic inequalities and diabetes in Iran $[25,44]$.

Although many studies indicate that smoking increases the risk of diabetes, some evidence supports the reverse association [45]. In our study, diabetes was more 
prevalent in ever-smokers, while for prediabetes a higher prevalence was observed among never-smokers. Also, studies have suggested that opium may temporarily decrease blood glucose [46]. Among people who had a history of opium use in the present study, the prevalence of prediabetes was slightly higher compared to those who had never used; however, for diabetes the prevalence was similar among both groups. Therefore, the association of cigarette and opium use with diabetes in the Iranian population needs further investigation.

\section{Limitations}

In this study, people above the age of 65 years were not included, which may have been led to a lower estimation of diabetes prevalence. Besides, using only one method for diagnosis and the unavailability of oral glucose tolerance test (OGTT) and HbA1c testing might be other causes of underestimating the prevalence of diabetes in this region of Iran. However, in many epidemiological studies in the region, there is significant discordance between OGTT and FBGbased prediabetes and diabetes diagnoses, and the prevalence of newly diagnosed diabetes is about 15 to $20 \%$ higher according to the FBG criteria [47]. The cross-sectional design and higher participation rate among women and housewives were among the other limitations of this study. Also, we were unable to exclude Type 1 diabetes from our analysis.

\section{Conclusions}

Prediabetes and diabetes are prevalent in southwestern Iran, and their prevalence is higher than in most parts of the country. The major determinants are older age, the presence of hypertension, and obesity. Further studies are needed to better understand the epidemiology of diabetes across Iran, and urgent interventions should be applied to escalate diabetes prevention, diagnosis, and treatment among high-risk populations residing in Iran.

\section{Abbreviations}

BMI: Body mass index; DBP: Diastolic blood pressure; DM: Diabetes mellitus; FBG: Fasting blood glucose; HbA1c: Hemoglobin A1c; HTN: Hypertension; KCHS: Khuzestan Comprehensive Health Study; MCA: Multiple correspondence analysis; MENA: Middle East and North Africa; NIMA D: National Institute for Medical Research Development; OGT: Oral glucose tolerance test; RR: Relative risk; SBP: Systolic blood pressure; SD: Standard deviation; WC: Waist circumference; WHO: World Health Organization

\section{Supplementary Information}

The online version contains supplementary material available at https://doi. org/10.1186/s12902-021-00790-x.

Additional file 1: Supplementary table. Distribution of people who were diagnosed with diabetes in the Khuzestan Comprehensive Health Study (KCHS), $n=4673$

\section{Acknowledgements}

This large population-based study was executed by the cooperation of Digestive Diseases Research Institute (DDRI), the Iranian Blood Transfusion Organization (IBTO), Jundishapur and Dezful Universities of Medical Sciences, together with Abadan and Behbahan Faculties of Medicine.

\section{Authors' contributions}

S.H. drafted the manuscript. Z.R. and ME.KH. contributed to interpretation of the analyses and drafting of the manuscript. Z.M supervised the project and was responsible for quality control. N.HM. thoroughly revised the manuscript before submission. A.KH. and S.M. performed the analysis. S.A. contributed to the literature review. SA.M., F.A., L.D., A.V., F.H., MM.MN., B.CH., and Y.P. contributed to data collection. H.P. and AA.SH. contributed to designing, supervising, and coordinating the project. All authors read and approved the final manuscript.

\section{Funding}

This study was funded by the National Institute for Medical Research Development (NIMAD) [grant number 940406].

\section{Availability of data and materials}

The datasets used and/or analysed during the current study are available from the corresponding author on reasonable request.

\section{Declarations}

\section{Ethics approval and consent to participate}

The ethics committee of the National Institute for Medical Research Development (NIMAD) has approved the study protocol (IR.NIMAD.REC.1394.002). Written informed consent was obtained from all participants.

\section{Consent for publication \\ Not Applicable.}

\section{Competing interests}

The authors declare that they have no competing interests.

\section{Author details}

${ }^{1}$ Liver and Pancreatobiliary Diseases Research Center, Digestive Diseases Research Institute, Shariati Hospital, Tehran University of Medical Sciences, N. Kargar St, Tehran, Iran. ${ }^{2}$ Hearing Research Center, Department of Biostatistics and Epidemiology, School of Public Health, Ahvaz Jundishapur University of Medical Sciences, Ahvaz, Iran. ${ }^{3}$ Endocrine Research Center, Institute of Endocrinology and Metabolism, Iran University of Medical Sciences, Tehran, Iran. ${ }^{4}$ Alimentary Tract Research Center, Imam Khomeini Hospital Clinical Research Development Unit, School of Medicine, Ahvaz Jundishapur University of Medical Sciences, Ahvaz, Iran. ${ }^{5}$ Faculty of Medicine, Behbahan University of Medical Sciences, Behbahan, Iran. ${ }^{6}$ Ahvaz Jundishapur University of Medical Sciences, Ahvaz, Iran. ${ }^{7}$ Shoushtar School of Medical Sciences, Shoushtar, Iran. ${ }^{8}$ Abadan Faculty of Medical Sciences, Abadan, Iran. ${ }^{9}$ Faculty of Medicine, Dezful University of Medical Sciences, Dezful, Iran. ${ }^{10}$ Digestive Diseases Research Institute, Tehran University of Medical Sciences, Tehran, Iran. ${ }^{11}$ Student Research Committee, Faculty of Paramedical Sciences, Shahid Beheshti University of Medical Sciences, Tehran, Iran. ${ }^{12}$ Alimentary Tract Research Center, Imam Khomeini Hospital Clinical Research Development Unit, Department of Biostatistics and Epidemiology, School of Public Health, Ahvaz Jundishapur University of Medical Sciences, Ahvaz, Iran. ${ }^{13}$ Alimentary Tract Research Center, Clinical Sciences Research Institute, Ahvaz Jundishapur University of Medical Sciences, Ahvaz, Iran.

Received: 10 January 2021 Accepted: 8 June 2021

Published online: 29 June 2021

\section{References}

1. Harati H, Hadaegh F, Saadat N, Azizi F. Population-based incidence of type 2 diabetes and its associated risk factors: results from a six-year cohort study in Iran. BMC Public Health. 2009;9(1):186. https://doi.org/10.1186/1471-24589-186. 
2. Shaghaghi A, Ahmadi A. Evidence gap on the prevalence of nonconventional risk factors for type 2 diabetes in Iran. Osong Public Health Res Perspect. 2014;5(5):292-7. https://doi.org/10.1016/j.phrp.2014.08.002.

3. Emdin CA, Anderson SG, Woodward M, Rahimi K. Usual blood pressure and risk of new-onset diabetes: evidence from 4.1 million adults and a metaanalysis of prospective studies. J Am Coll Cardiol. 2015;66(14):1552-62. https://doi.org/10.1016/j.jacc.2015.07.059.

4. Guariguata L, Whiting DR, Hambleton I, Beagley J, Linnenkamp U, Shaw JE. Global estimates of diabetes prevalence for 2013 and projections for 2035 Diabetes Res Clin Pract. 2014;103(2):137-49. https://doi.org/10.1016/j.dia bres.2013.11.002.

5. Federation ID. IDF Diabetes Atlas. 6th ed; 2013

6. Al Busaidi N, Shanmugam P, Manoharan D. Diabetes in the Middle East: government health care policies and strategies that address the growing diabetes prevalence in the Middle East. Curr Diabetes Rep. 2019;19(2):8. https://doi.org/10.1007/s11892-019-1125-6.

7. Collaboration NCDRF. Worldwide trends in diabetes since 1980: a pooled analysis of 751 population-based studies with 4.4 million participants. Lancet (London, England). 2016;387(10027):1513-30.

8. Javanbakht M, Mashayekhi A, Baradaran HR, Haghdoost A, Afshin A. Projection of Diabetes Population Size and Associated Economic Burden through 2030 in Iran: Evidence from Micro-Simulation Markov Model and Bayesian Meta-Analysis. PLoS One. 2015;10(7):e0132505-e.

9. Moosazadeh M, Afshari M, Jafari K, Kheradmand M, Kashi Z, Aarabi M, et al. Enrolment phase results of the Tabari cohort study: comparing family history, lipids and anthropometric profiles among diabetic patients. Osong Public Health Res Perspect. 2019;10(5):289-94. https://doi.org/10.24171/j. phrp.2019.10.5.05.

10. Sarayani A, Rashidian A, Gholami K. Low utilisation of diabetes medicines in Iran, despite their affordability (2000-2012): a time-series and benchmarking study. BMJ Open. 2014;4(10):e005859. https://doi.org/10.1136/bmjopen-2 014-005859.

11. Zarei L, Peymani P, Moradi N, Kheirandish M, Mirjalili M, Zare M. Affordability of Medication Therapy in Diabetic Patients: A Scenario-Based Assessment in Iran's Health System Context. Int J Health Policy Manag. 2020. https://doi. org/10.34172/ijhpm.2020.152. Epub ahead of print. PMID: 32861229.

12. Esteghamati A, Larijani B, Aghajani MH, Ghaemi F, Kermanchi J, Shahrami A, et al. Diabetes in Iran: Prospective Analysis from First Nationwide Diabetes Report of National Program for Prevention and Control of Diabetes (NPPCD2016). Sci Rep. 2017;7(1):13461.

13. Jafarian-Amirkhizi A, Sarayani A, Gholami K, Taghizadeh-Ghehi M, Heidari K, Jafarzadeh-Kohneloo A, et al. Adherence to medications, self-care activity, and $\mathrm{HbA} 1 \mathrm{c}$ status among patients with type 2 diabetes living in an urban area of Iran. J Diabetes Metab Disord. 2018;17(2):165-72. https://doi.org/10.1 007/s40200-018-0356-4.

14. Farzadfar F, Murray CJ, Gakidou E, Bossert T, Namdaritabar H, Alikhani S, et al. Effectiveness of diabetes and hypertension management by rural primary health-care workers (Behvarz workers) in Iran: a nationally representative observational study. Lancet (London, England). 2012; 379(9810):47-54.

15. Noshad S, Afarideh M, Heidari B, Mechanick JI, Esteghamati A. Diabetes Care in Iran: where we stand and where we are headed. Ann Glob Health. 2015; 81(6):839-50. https://doi.org/10.1016/j.aogh.2015.10.003.

16. Faraji O, Etemad K, Akbari Sari A, Ravaghi H. Policies and programs for prevention and control of diabetes in Iran: a document analysis. Global J Health Sci. 2015;7(6):187-97. https://doi.org/10.5539/gjhs.v7n6p187.

17. Cheraghian B, Sharafkhah M, Mohammadi Z, Hariri S, Rahimi Z, Danehchin L, et al. The Khuzestan comprehensive health study (KCHS): methodology and profile of participants. Arch Iranian Med. 2020;23(10):653-7. https://doi.org/1 0.34172/aim.2020.82

18. WHO Expert Consultation. Appropriate body-mass index for Asian populations and its implications for policy and intervention strategies. Lancet. 2004;363(9403):157-63. https://doi.org/10.1016/S0140-6736(03)152 68-3. Erratum in: Lancet. 2004 Mar 13;363(9412):902. PMID: 14726171.

19. Azizi F, Khalili D, Aghajani H, Esteghamati A, Hosseinpanah F, Delavari A, et al. Appropriate waist circumference cut-off points among Iranian adults: the first report of the Iranian National Committee of obesity. Arch Iranian Med. 2010;13(3):243-4.

20. Armstrong C. High blood pressure: ACC/AHA releases updated guideline. Am Fam Physician. 2018;97(6):413-5.

21. Johnson RA, Wichern DW. Applied multivariate statistical analysis. 2002;5(8).
22. Ebrahimi $\mathrm{H}$, Emamian $\mathrm{MH}$, Hashemi $\mathrm{H}$, Fotouhi A. High incidence of diabetes mellitus among a middle-aged population in Iran: a longitudinal study. Can J Diabetes. 2016;40(6):570-5. https://doi.org/10.1016/j.jcjd.2016. 05.012.

23. Rahmanian K, Shojaei M, Sotoodeh Jahromi A, Madani A. The association between pre-diabetes with body mass index and marital status in an Iranian urban population. Global J Health Sci. 2015;8(4):95-101. https://doi.org/10. 5539/gjhs.v8n4p95.

24. Yazdanpanah L, Shahbazian HB, Moravej Aleali A, Jahanshahi A, Ghanbari S, Latifi SM. Prevalence, awareness and risk factors of diabetes in Ahvaz (south west of Iran). Diabetes Metab Syndr. 2016;10(2 Suppl 1):S114-8. https://doi. org/10.1016/j.dsx.2016.03.007

25. Mirzaei M, Rahmaninan M, Mirzaei M, Nadjarzadeh A, Dehghani Tafti AA. Epidemiology of diabetes mellitus, pre-diabetes, undiagnosed and uncontrolled diabetes in Central Iran: results from Yazd health study. BMC Public Health. 2020;20(1):166

26. Esteghamati A, Meysamie A, Khalilzadeh O, Rashidi A, Haghazali M, Asgari F, et al. Third national Surveillance of Risk Factors of Non-Communicable Diseases (SURFNCD-2007) in Iran: methods and results on prevalence of diabetes, hypertension, obesity, central obesity, and dyslipidemia. BMC Public Health. 2009;9:167.

27. Safari-Faramani R, Rajati F, Tavakol K, Hamzeh B, Pasdar Y, Moradinazar M, et al. Prevalence, awareness, treatment, control, and the associated factors of diabetes in an Iranian Kurdish population. J Diabetes Res. 2019;2019: 5869206.

28. Najafipour H, Sanjari M, Shokoohi M, Haghdoost A-A, Afshari M, Shadkam M, et al. Epidemiology of diabetes mellitus, pre-diabetes, undiagnosed and uncontrolled diabetes and its predictors in general population aged 15 to 75 years: a community-based study (KERCADRS) in southeastern Iran. J Diabetes. 2015;7(5):613-21. https://doi.org/10.1111/1753-0407.12195.

29. Akbarzadeh A, Salehi A, Molavi Vardanjani H, Poustchi H, Gandomkar A, Fattahi MR, et al. Epidemiology of adult diabetes mellitus and its correlates in pars cohort study in southern Iran. Arch Iranian Med. 2019;22(11):633-9.

30. Chentli F, Azzoug S, Mahgoun S. Diabetes mellitus in elderly. Indian J Endocrinol Metab. 2015;19(6):744-52. https://doi.org/10.4103/2230-8210.1 67553.

31. Honarvar B, Banakar M, Hassani N, Movahednezhad Y, Gheibi Z, Bagheri LK. From Iceberg of Pre-diabetes to Poor Glycemic Control in Diabetics: An Elderly Based Study in Shiraz, South of Iran. Int J Prev Med. 2019;10:171.

32. Kautzky-Willer A, Harreiter J, Pacini G. Sex and gender differences in risk, pathophysiology and complications of type 2 diabetes mellitus. Endocr Rev. 2016;37(3):278-316. https://doi.org/10.1210/er.2015-1137.

33. Zhang H, Ni J, Yu C, et al. Sex-Based Differences in Diabetes Prevalence and Risk Factors: A Population-Based Cross-Sectional Study Among Low-Income Adults in China. Front Endocrinol. 2019;10:658. https://doi.org/10.3389/ fendo.2019.00658. Published 2019 Sep 25

34. Siddiqui S, Zainal H, Harun SN, Sheikh Ghadzi SM, Ghafoor S. Gender differences in the modifiable risk factors associated with the presence of prediabetes: a systematic review. Diabetes Metab Syndr. 2020;14(5):1243-52. https://doi.org/10.1016/j.dsx.2020.06.069.

35. Barzin M, Asghari G, Hosseinpanah F, Mirmiran P, Azizi F. The association of anthropometric indices in adolescence with the occurrence of the metabolic syndrome in early adulthood: Tehran lipid and glucose study (TLGS). Pediatric Obesity. 2013;8(3):170-7. https://doi.org/10.1111/j.2047-631 0.2012.00102.x.

36. Logue J, Walker JJ, Colhoun HM, Leese GP, Lindsay RS, McKnight JA, et al. Do men develop type 2 diabetes at lower body mass indices than women? Diabetologia. 2011;54(12):3003-6. https://doi.org/10.1007/ s00125-011-2313-3.

37. Namayandeh SM, Karimi A, Fallahzadeh H, Rahmanian M, Sadr Bafghi SM, Soltani $M$, et al. The incidence rate of diabetes mellitus (type II) and its related risk factors: a 10-year longitudinal study of Yazd healthy Heart cohort (YHHC), Iran. Diabetes Metab Syndr. 2019;13(2):1437-41. https://doi. org/10.1016/j.dsx.2019.02.012.

38. Mirzaei M, Khajeh M, Askarishahi M, Azizi R. Behavioral and familial predictors of diabetes mellitus in adults aged 20-69 in Yazd, Iran during 2014-2015. Diabetes Metab Syndr. 2018;12(5):667-71. https://doi.org/10.101 6/j.dsx.2018.04.002.

39. Najafipour M, Zareizadeh M, Najafipour F. Epidemiologic study of familial type 2 diabetes in Tehran. J Adv Pharm Technol Res. 2018;9(2):56-60. https://doi.org/10.4103/japtr.JAPTR_228_18. 
40. Rashedi V, Asadi-Lari M, Delbari A, Fadayevatan R, Borhaninejad V, Foroughan M. Prevalence of diabetes type 2 in older adults: findings from a large population-based survey in Tehran, Iran (urban HEART-2). Diabetes Metab Syndr. 2017;11(Suppl 1):S347-s50. https://doi.org/10.1016/.dsx.2017. 03.014 .

41. Derakhshan A, Sardarinia M, Khalili D, Momenan AA, Azizi F, Hadaegh F. Sex specific incidence rates of type 2 diabetes and its risk factors over 9 years of follow-up: Tehran lipid and glucose study. PLoS One. 2014;9(7):e102563. https://doi.org/10.1371/journal.pone.0102563.

42. Khosravi A, Emamian MH, Hashemi H, Fotouhi A. Obesity is the Most Important Factor for Gender Inequality in Type 2 Diabetes Incidence in an Iranian Population. Int J Prev Med. 2019;10:215.

43. Golozar A, Khademi H, Kamangar F, Poutschi H, Islami F, Abnet CC, et al. Diabetes mellitus and its correlates in an Iranian adult population. PLOS One. 2011:6(10):e26725. https://doi.org/10.1371/journal.pone.0026725.

44. Peykari N, Djalalinia S, Qorbani M, Sobhani S, Farzadfar F, Larijani B. Socioeconomic inequalities and diabetes: A systematic review from Iran. J Diabetes Metab Disord. 2015;14:8.

45. Yeh HC, Duncan BB, Schmidt MI, Wang NY, Brancati FL. Smoking, smoking cessation, and risk for type 2 diabetes mellitus: a cohort study. Ann Intern Med. 2010;152(1):10-7. https://doi.org/10.7326/0003-4819-152-1-20100105000005.

46. Azod L, Rashidi M, Afkhami-Ardekani M, Kiani G, Khoshkam F. Effect of opium addiction on diabetes. Am J Drug and Alcohol Abuse. 2008;34(4): 383-8. https://doi.org/10.1080/00952990802122580.

47. Kumar R, Nandhini LP, Kamalanathan S, Sahoo J, Vivekanadan M. Evidence for current diagnostic criteria of diabetes mellitus. World J Diabetes. 2016; 7(17):396-405. https://doi.org/10.4239/wjd.v7.117.396.

\section{Publisher's Note}

Springer Nature remains neutral with regard to jurisdictional claims in published maps and institutional affiliations.

Ready to submit your research? Choose BMC and benefit from:

- fast, convenient online submission

- thorough peer review by experienced researchers in your field

- rapid publication on acceptance

- support for research data, including large and complex data types

- gold Open Access which fosters wider collaboration and increased citations

- maximum visibility for your research: over $100 \mathrm{M}$ website views per year

At $\mathrm{BMC}$, research is always in progress.

Learn more biomedcentral.com/submissions 